\title{
A MULTIPLICITY RESULT FOR A CLASS OF EQUATIONS OF $p$-LAPLACIAN TYPE WITH SIGN-CHANGING NONLINEARITIES
}

\author{
NGUYEN THANH CHUNG \\ Department of Mathematics and Informatics, Quang Binh University, 312 Ly Thuong Kiet, \\ Dong Hoi, Quang Binh, Vietnam \\ e-mail: ntchung82@yahoo.com \\ and QUỐC ANH NGÔ \\ Department of Mathematics, College of Science, Vietnam National University, Hanoi, Vietnam \\ Department of Mathematics, National University of Singapore, Science Drive 2, Singapore 117543 \\ e-mail:bookworm_vn@yahoo.com
}

(Received 11 July 2008; accepted 22 December 2008)

Abstract. Using variational arguments we study the non-existence and multiplicity of non-negative solutions for a class equations of the form

$$
-\operatorname{div}(a(x, \nabla u))=\lambda f(x, u) \text { in } \Omega,
$$

where $\Omega$ is a bounded domain in $\mathbb{R}^{N}, N \geqq 3, f$ is a sign-changing Carathéodory function on $\Omega \times[0,+\infty)$ and $\lambda$ is a positive parameter.

2002 Mathematics Subject Classification. 35J20, 35J60, 35J65, 58 E05.

1. Introduction. This paper deals with the non-existence and multiplicity of non-negative, non-trivial solutions to the following problem,

$$
\begin{aligned}
-\operatorname{div}(a(x, \nabla u)) & =\lambda f(x, u) \text { in } \Omega, \\
u & =0 \text { on } \partial \Omega,
\end{aligned}
$$

where $\Omega$ is a bounded domain in $\mathbb{R}^{N}$, function $a$ satisfies

$$
|a(x, \xi)| \leqq c_{0}\left(h_{0}(x)+h_{1}(x)|\xi|^{p-1}\right)
$$

for all $\xi \in \mathbb{R}^{N}$, a.e. $x \in \Omega, h_{0}(x) \geqq 0, h_{1}(x) \geqq 1$ for a.e. $x \in \Omega, p \geqq 2$ and $\lambda>0$ is a parameter. When $h_{0}$ and $h_{1}$ belong to $L^{\infty}(\Omega)$, the problem has been studied by many authors (see $[2, \mathbf{8}, \mathbf{1 0}]$ for details). Here we study the situation that $h_{0} \in L^{\frac{p}{p-1}}(\Omega)$ and $h_{1} \in L_{l o c}^{1}(\Omega)$. Then problem (1.1)-(1.2) now may be non-uniform in the sense that the functional associated to the problem may be infinity for some $u$.

We point out the fact that in $[4,13]$, D. M. Duc and N. T. Vu have studied the following Dirichlet elliptic problem,

$$
\begin{aligned}
-\operatorname{div}(a(x, \nabla u)) & =f(x, u) \text { in } \Omega, \\
u & =0 \text { on } \partial \Omega,
\end{aligned}
$$

where the nonlinear term $f$ verifies the so-called Ambrosetti-Rabinowitz condition. The authors obtained the existence of a weak solution by using a variant of the 
Mountain pass theorem introduced in [3]. Then, H. Q. Toan and Q.-A. Ngô [12] gave some multiplicity results in the case when $f(x, u)=h(x)|u|^{r-1} u+g(x)|u|^{s-1} u$. Using the Mountain pass theorem in [3] combined with Ekeland's variational principle in [5] they proved that problem (1.3)-(1.4) has at least two weak solutions.

Motivated by K. Perera [10] and M. Mihăilescu and V. Rădulescu [7], the goal of this work is to investigate the problem (1.1)-(1.2) with positive parameter $\lambda$ and the sign-changing nonlinearity $f$. We also do not require that the nonlinear term $f$ verifies the Ambrosetti-Rabinowitz condition as in $[4, \mathbf{1 2}]$.

In order to state our main result, let us introduce the following hypotheses on problem (1.1)-(1.2).

Assume that $N \geqq 3$ and $2 \leqq p<N$. Let $\Omega$ be a bounded domain with a smooth boundary $\partial \Omega$. Consider that $a: \Omega \times \mathbb{R}^{N} \rightarrow \mathbb{R}^{N}, a=a(x, \xi)$, is the continuous derivative with respect to $\xi$ of the continuous function $A: \Omega \times \mathbb{R}^{N} \rightarrow \mathbb{R}, A=A(x, \xi)$, that is, $a(x, \xi)=\partial A(x, \xi) / \partial \xi$ and $A(x, 0)=0$ for a.e. $x \in \Omega$. Assume that there are positive constant $c_{0}$ and two non-negative measurable functions $h_{0}, h_{1}$ such that $h_{0} \in L^{p / p-1}(\Omega), h_{1} \in L_{l o c}^{1}(\Omega), h_{1}(x) \geqq 1$, a.e. $x \in \Omega$. Suppose that $a$ and $A$ satisfy the following hypotheses.

(A) $|a(x, \xi)| \leqq c_{0}\left(h_{0}(x)+h_{1}(x)|\xi|^{p-1}\right)$ for all $\xi \in \mathbb{R}^{N}$, a.e. $x \in \Omega$.

(A) The following inequality holds

$$
0 \leqq(a(x, \xi)-a(x, \psi)) \cdot(\xi-\psi)
$$

for all $\xi, \psi \in \mathbb{R}^{N}$, a.e. $x \in \Omega$, with equality if and only if $\xi=\psi$.

$\left(\mathbf{A}_{3}\right)$ There exists a positive constant $k_{0}$ such that

$$
A\left(x, \frac{\xi+\psi}{2}\right) \leqq \frac{1}{2} A(x, \xi)+\frac{1}{2} A(x, \psi)-k_{0} h_{1}(x)|\xi-\psi|^{p}
$$

for all $\xi, \psi \in \mathbb{R}^{N}$, a.e. $x \in \Omega$, that is, $A$ is $p$-uniformly convex.

(A4) There exists a constant $k_{1}>0$ such that the following inequalities hold true

$$
k_{1} h_{1}(x)|\xi|^{p} \leqq a(x, \xi) \cdot \xi \leqq p A(x, \xi)
$$

for all $\xi \in \mathbb{R}^{N}$, a.e. $x \in \Omega$.

EXAMPLE 1.

1. Let

$$
A(x, \xi)=\frac{h(x)}{p}|\xi|^{p}, \quad a(x, \xi)=h(x)|\xi|^{p-2} \xi
$$

with $p \geqq 2$ and $h \in L_{l o c}^{1}(\Omega)$. Then we get the operator $\operatorname{div}\left(h(x)|\nabla u|^{p-2} \nabla u\right)$, and if $h(x) \equiv 1$ in $\Omega$ we conclude the well-known $p$-Laplacian operator

$$
\Delta_{p} u:=\operatorname{div}\left(|\nabla u|^{p-2} \nabla u\right)
$$

2. Let

as in $[\mathbf{8}, \mathbf{1 0}]$.

$$
A(x, \xi)=\frac{h(x)}{p}\left(\left(1+|\xi|^{2}\right)^{\frac{p}{2}}-1\right)
$$


with $p \geqq 2, h \in L^{\frac{p}{p-1}}(\Omega)$. Then

$$
a(x, \xi)=h(x)\left(1+|\xi|^{2}\right)^{\frac{p-2}{2}} \xi .
$$

We obtain the generalised mean curvature operator

$$
\operatorname{div}\left(h(x)\left(1+|\nabla u|^{2}\right)^{\frac{p-2}{2}} \nabla u\right) .
$$

It should be observed that the above examples have not been considered in $[\mathbf{2 , 8 , 1 0}$ yet. For more information and connection on these operators, the reader may consult either [2] or [8] and the references therein.

As in [10], we assume that function $f: \Omega \times[0,+\infty) \rightarrow \mathbb{R}$ is a sign-changing Carathéodory function and satisfies the following hypotheses:

(F $\left.\mathbf{F}_{1}\right) f(x, 0)=0,|f(x, t)| \leqq C t^{p-1}$ for all $t \in[0 .+\infty)$, a.e. $x \in \Omega$, and for some constant $C>0$.

$\left(\mathbf{F}_{2}\right)$ There exist two positive constants $t_{0}, t_{1}>0$ such that $F(x, t) \leqq 0$ for $0 \leqq t \leqq t_{0}$ and $F\left(x, t_{1}\right)>0$.

(F) $\limsup _{t \rightarrow \infty} \frac{F(x, t)}{t^{p}} \leqq 0$ uniformly in $x$, where $F(x, t)=\int_{0}^{t} f(x, s) \mathrm{d} s$.

Let $W^{1, p}(\Omega)$ be the usual Sobolev space and $W_{0}^{1, p}(\Omega)$ be the closure of $C_{0}^{\infty}(\Omega)$ under the norm

$$
\|u\|=\left(\int_{\Omega}|\nabla u|^{p} \mathrm{~d} x\right)^{\frac{1}{p}}
$$

We now consider the following subspace of $W_{0}^{1, p}(\Omega)$ :

$$
H:=\left\{u \in W_{0}^{1, p}(\Omega): \int_{\Omega} h_{1}(x)|\nabla u|^{p} \mathrm{~d} x<+\infty\right\} .
$$

Then $H$ is an infinite dimensional Banach space with respect to the norm (see [4])

$$
\|u\|_{H}=\left(\int_{\Omega} h_{1}(x)|\nabla u|^{p} \mathrm{~d} x\right)^{\frac{1}{p}} .
$$

We define the functional $\Phi_{\lambda}: H \rightarrow \mathbb{R}$ by

$$
\Phi_{\lambda}(u)=\Lambda(u)-I(u),
$$

where

$$
\Lambda(u)=\int_{\Omega} A(x, \nabla u) \mathrm{d} x, \quad I(u)=\lambda \int_{\Omega} F(x, u) \mathrm{d} x, \quad u \in H .
$$

Since $h_{0} \in L^{p / p-1}(\Omega)$, then the value $\Phi_{\lambda}(u)$ may be infinity for some $u \in W_{0}^{1, p}(\Omega)$, that is, the functional may not be defined throughout $W_{0}^{1, p}(\Omega)$. In order to overcome this difficulty, we choose the subspace $H$ of $W_{0}^{1, p}(\Omega)$. 
only if

DEFINITION 1. We say that $u \in H$ is a weak solution of problem (1.1)-(1.2) if and

$$
\int_{\Omega} a(x, \nabla u) \nabla \varphi \mathrm{d} x-\lambda \int_{\Omega} f(x, u) \varphi \mathrm{d} x=0
$$

for all $\varphi \in C_{0}^{\infty}(\Omega)$.

Then we have the following remark which plays an important role in our arguments.

REMARK 1.

(i) By $\left(A_{4}\right)$ and (i) in Proposition 2, it is easy to see that

$$
H=\left\{u \in W_{0}^{1, p}(\Omega): \Lambda(u)<\infty\right\}=\left\{u \in W_{0}^{1, p}(\Omega): \Phi_{\lambda}(u)<\infty\right\} .
$$

(ii) Since $h_{1}(x) \geqq 1$, a.e. $x \in \Omega$, we have $\|u\| \leqq\|u\|_{H}$ for all $u \in H$. Thus, the continuous embeddings

$$
H \hookrightarrow W_{0}^{1, p}(\Omega) \hookrightarrow L^{i}(\Omega), \quad p \leqq i \leqq p^{*}
$$

hold true.

(iii) Since $\int_{\Omega} h_{1}(x)|\nabla u|^{p} \mathrm{~d} x<+\infty$ for any $u \in C_{0}^{\infty}(\Omega)$ and $h_{1} \in L_{l o c}^{1}(\Omega)$, we have $C_{0}^{\infty}(\Omega) \subset H$.

The main result for the existence of solutions of (1.1) can be formulated as follows.

THEOREM 1. Under hypotheses $\left(\mathrm{A}_{1}\right)-\left(\mathrm{A}_{4}\right)$ and $\left(\mathrm{F}_{1}\right)$, there exists a positive constant $\underline{\lambda}$ such that for all $\lambda \in(0, \underline{\lambda})$, problem $(1.1)-(1.2)$ has no weak solution.

THEOREM 2. Under hypotheses $\left(\mathrm{A}_{1}\right)-\left(\mathrm{A}_{4}\right)$ and $\left(\mathrm{F}_{1}\right)-\left(\mathrm{F}_{3}\right)$, there exists a positive constant $\bar{\lambda}$ such that for all $\lambda \geqq \bar{\lambda}$, problem (1.1)-(1.2) has at least two distinct nonnegative, non-trivial weak solutions.

To prove Theorem 2, we first prove that the functional associated to the problem (1.1)-(1.2) is bounded from below and coercive, and thus the first weak solution is obtained due to a variant of the minimum principle which we will prove in the next section (see Theorem 4). To obtain the second solution to the problem (1.1)-(1.2), we shall use a variant of the mountain pass theorem due to Duc (see Proposition 1).

2. Auxiliary results. Due to the presence of $h_{1}$, functional $\Lambda$ may not be continuously Fréchet differentiable functionals on $H$. This means that we cannot apply the classical Mountain pass theorem by Ambrosetti-Rabinowitz (see [1] for details). To overcome this difficulty, we shall use a weak version of the Mountain pass theorem introduced by Duc [3]. Now we introduce the following concept of weakly continuously differentiability due to Duc.

Definition 2. Let $\mathcal{F}$ be a map from a Banach space $X$ to $\mathbb{R}$. We say that $\mathcal{F}$ is weakly continuously differentiable on $X$ if and only if the following two conditions are satisfied:

(i) For any $u \in X$ there exists a linear map $D \mathcal{F}(u)$ from $X$ to $\mathbb{R}$ such that

$$
\lim _{t \rightarrow 0} \frac{\mathcal{F}(u+t v)-\mathcal{F}(u)}{t}=D \mathcal{F}(u)(v)
$$

for every $v \in X$. 
(ii) For any $v \in X$, the map $u \mapsto D \mathcal{F}(u)(v)$ is continuous on $X$.

REMARK 2. If we suppose further that $v \mapsto D \mathcal{F}(u)(v)$ is continuous linear mapping on $X$, then $\mathcal{F}$ is Gâteaux differentiable.

DEFINITION 3. We call $u$ a generalised critical point (critical point, for short) of $\mathcal{F}$ if $D \mathcal{F}(u)=0 . c$ is called a generalised critical value (critical value, for short) of $\mathcal{F}$ if $\mathcal{F}(u)=c$ for some critical point $u$ of $\mathcal{F}$.

Denote by $C_{w}^{1}(X)$ the set of weakly continuously differentiable functionals on $X$. It is clear that $C^{1}(X) \subset C_{w}^{1}(X)$ where we denote by $C^{1}(X)$ the set of all continuously Fréchet differentiable functionals on $X$. Now let $\mathcal{F} \in C_{w}^{1}(X)$; we put

$$
\|D \mathcal{F}(u)\|=\sup \{|D \mathcal{F}(u)(h)| h \in Y,\|h\|=1\}
$$

for any $u \in X$, where $\|D \mathcal{F}(u)\|$ may be $+\infty$.

Definition 4. We say that $\mathcal{F}$ satisfies the Palais-Smale condition at level $c \in \mathbb{R}$ (denoted by $(\mathrm{PS})_{c}$ ) if any sequence $\left\{u_{n}\right\} \subset X$ for which

$$
\mathcal{F}\left(u_{n}\right) \rightarrow c \quad \text { and } \quad D \mathcal{F}\left(u_{n}\right) \rightarrow 0 \text { in } X^{\star}
$$

possesses a convergent subsequence. If this is true at every level $c$ then we simply say that $\mathcal{F}$ satisfies the Palais-Smale condition (denoted by (PS)).

Definition 5. We say that $\mathcal{F}$ satisfies the Cerami condition at level $c \in \mathbb{R}$ (denoted by $\left.(\mathrm{C})_{c}\right)$ if any sequence $\left\{u_{n}\right\} \subset X$ for which

$$
\mathcal{F}\left(u_{n}\right) \rightarrow c \quad \text { and } \quad\left(1+\left\|x_{n}\right\|\right) D \mathcal{F}\left(u_{n}\right) \rightarrow 0 \text { in } X^{\star}
$$

possesses a convergent subsequence. If this is true at every level $c$, then we simply say that $\mathcal{F}$ satisfies the Cerami condition (denoted by $(\mathrm{C})$ ).

In the proof of our main theorems, we shall use the following results which is proved in [9]. We will recall its proof for completeness.

TheOrEm 3 (see [9]). Let $\mathcal{F} \in C_{w}^{1}(X)$ where $X$ is a Banach space. Assume that

(i) $\mathcal{F}$ is bounded from below, $c=\inf \mathcal{F}$,

(ii) $\mathcal{F}$ satisfies the (PS) condition.

Then c is a critical value of $\mathcal{F}$ (i.e. there exists a critical point $u_{0} \in X$ such that $\mathcal{F}\left(u_{0}\right)=c$ )

Proof of Theorem 3. Let $c$ be an arbitrary real number. Before proving the theorem, we need the following notation:

$$
\mathcal{F}^{c}=\{u \in X \mid \mathcal{F}(u) \leqq c\} .
$$

Let us assume, by negation, that $c$ is not a critical value of $\mathcal{F}$. Then, Theorem 2.2 in [13] implies the existence of $\varepsilon>0$ and $\eta \in C([0,+\infty) \times X, X)$ satisfying $\eta\left(1, \mathcal{F}^{c+\varepsilon}\right) \subset \mathcal{F}^{c-\varepsilon}$. This is a contradiction since $\mathcal{F}^{c-\varepsilon}=\emptyset$ due to the fact that $c=\inf \mathcal{F}$.

REMARK 3. By Corollary 2.1.1 in [6], if $\mathcal{F}: X \rightarrow \mathbb{R}$ is a locally Lipschitz, bounded from below function and it satisfies the $(\mathrm{C})$ condition, then $\mathcal{F}$ is coercive. This leads us to state the following lemma. 
LEMMA 1. If $\mathcal{F}: X \rightarrow \mathbb{R}$ is a locally Lipschitz, bounded from below function and it satisfies the $(C)$ condition then it satisfies the (PS) condition.

Proof. Let $\left\{u_{n}\right\}_{n} \subset X$ be a sequence such that $\mathcal{F}\left(u_{n}\right)$ is bounded and $D \mathcal{F}\left(u_{n}\right) \rightarrow 0$ in $X^{\star}$. By Remark $3, \mathcal{F}$ is coercive, and this helps us to deduce that $\left\{u_{n}\right\}_{n}$ is bounded in $X$. Hence also $\left(1+\left\|u_{n}\right\|\right) D \mathcal{F}\left(u_{n}\right) \rightarrow 0$ in $X^{\star}$, and because $\mathcal{F}$ satisfies the $(\mathrm{C})$ condition, it follows that $\left\{u_{n}\right\}_{n}$ has a strongly convergent subsequence. This completes the proof.

Similar to Theorem 3, we have the following new result.

THeOREM 4. Let $\mathcal{F}$ be continuous on $X$ and be of class $C_{w}^{1}(X)$ where $X$ is a Banach space. Assume that

(i) $\mathcal{F}$ is bounded from below, $c=\inf \mathcal{F}$,

(ii) $\mathcal{F}$ satisfies the $(C)$ condition.

Then $c$ is a critical value of $\mathcal{F}$ (i.e. there exists a critical point $u_{0} \in X$ such that $\mathcal{F}\left(u_{0}\right)=c$ ).

The proof of Theorem 4 follows from Lemma 1, so we omit it. Next we provide a variant Mountain pass theorem due to Duc [3].

Proposition 1 (see [3]). Let $\mathcal{F} \in C_{w}^{1}(X)$ where $X$ is a Banach space and satisfies (PS) condition. Assume that $\mathcal{F}(0)=0$ and there exist a positive constant $\rho$ and $z_{0} \in X$ such that

(i) $\left\|z_{0}\right\|_{X}>\rho$ and $\mathcal{F}\left(z_{0}\right) \leqq 0$.

(ii) $\alpha=\inf \left\{\mathcal{F}(u): u \in X,\|u\|_{X}=\rho\right\}>0$.

Assume that the set

$$
G=\left\{\varphi \in C([0,1], X): \varphi(0)=0, \varphi(1)=z_{0}\right\}
$$

is not empty. Put

$$
\beta:=\inf \{\max \mathcal{F}(\varphi([0,1])): \varphi \in G\} .
$$

Then $\beta \geqq \alpha$ and $\beta$ is a critical value of $\mathcal{F}$.

For the use of Proposition 1, we refer the reader to $[3, \mathbf{1 2}, \mathbf{1 3}]$. We end this section by studying some certain properties of the functional $\Phi_{\lambda}$ given by (1.5) but we first recall some results which will be used throughout this work.

Proposition 2 (see [4]).

(i) A verifies the growth condition

$$
|A(x, \xi)| \leqq c_{0}\left(h_{0}(x)|\xi|+h_{1}(x)|\xi|^{p}\right)
$$

for all $\xi \in \mathbb{R}^{N}$, a.e. $x \in \Omega$.

(ii) $A(x, \xi)$ is convex with respect to $\xi$. Moreover, by $\left(A_{3}\right)$ for all $u, v \in H$ we have

$$
\Lambda\left(\frac{u+v}{2}\right) \leqq \frac{1}{2} \Lambda(u)+\frac{1}{2} \Lambda(v)-k_{0}\|u-v\|_{H}^{p} .
$$

Using the method as in [4] with some simple computations we obtain the following proposition which concerns the smoothness of the functional $\Phi_{\lambda}$. 
Proposition 3.

(i) If $\left\{u_{m}\right\}$ is a sequence weakly converging to $u$ in $W_{0}^{1, p}(\Omega)$, then

$$
\Lambda(u) \leqq \liminf _{m \rightarrow \infty} \Lambda\left(u_{m}\right)
$$

and

$$
\lim _{m \rightarrow \infty} I\left(u_{m}\right)=I(u)
$$

(ii) The functionals $\Lambda$ and I are continuous on $H$.

(iii) Functional $\Phi_{\lambda}$ is weakly continuously differentiable on $H$ and we have

$$
D \Phi_{\lambda}(u)(\varphi)=\int_{\Omega} a(x, \nabla u) \nabla \varphi \mathrm{d} x-\lambda \int_{\Omega} f(x, u) \varphi \mathrm{d} x
$$

for all $u, \varphi \in H$.

\section{Proofs of the theorems.}

Proof of Theorem 1. Let us denote by $S$ the best constant in the Sobolev embedding $W_{0}^{1, p}(\Omega) \hookrightarrow L^{p}(\Omega)$, i.e.

$$
S=\inf _{W_{0}^{1, p}(\Omega) \backslash\{0\}} \frac{\left(\int_{\Omega}|\nabla u|^{p} \mathrm{~d} x\right)^{\frac{1}{p}}}{\left(\int_{\Omega}|u|^{p} \mathrm{~d} x\right)^{\frac{1}{p}}} .
$$

Then, if $u$ is a weak solution of problem (1.1)-(1.2), multiplying (1.1) by $u$ and integrating by parts combined with conditions $\left(\mathrm{A}_{4}\right)$ and $\left(\mathrm{F}_{1}\right)$ gives

$$
\begin{aligned}
k_{1} \int_{\Omega}|\nabla u|^{p} \mathrm{~d} x & \leqq k_{1} \int_{\Omega} h_{1}(x)|\nabla u|^{p} \mathrm{~d} x \\
& \leqq \int_{\Omega} a(x, \nabla u) \nabla u \mathrm{~d} x=\lambda \int_{\Omega} f(x, u) u \mathrm{~d} x \leqq C \lambda \int_{\Omega}|u|^{p} \mathrm{~d} x .
\end{aligned}
$$

Hence, choosing $\underline{\lambda}=k_{1} S / C$, where $S$ is given by (3.1), we conclude the proof.

We will prove Theorem 2 by using critical point theory. Set $f(x, t)=0$ for all $t<0$ and consider the energy functional $\Phi_{\lambda}: H \rightarrow \mathbb{R}$ which is given by (1.5).

LEMMA 2. If $u$ is a critical point of $\Phi_{\lambda}$ then $u$ is non-negative in $\Omega$.

Proof. Observe that if $u$ is a critical point of $\Phi_{\lambda}$, denoting by $u^{-}$the negative part of $u$, i.e. $u^{-}(x)=\min \{u(x), 0\}$ we have

$$
\begin{aligned}
0=D \Phi_{\lambda}(u)\left(u^{-}\right) & =\int_{\Omega} a(x, \nabla u) \nabla u^{-} \mathrm{d} x-\lambda \int_{\Omega} f(x, u) u^{-} \mathrm{d} x \\
& \geqq k_{1} \int_{\Omega} h_{1}(x)\left|\nabla u^{-}\right|^{p} \mathrm{~d} x=k_{1}\left\|u^{-}\right\|_{H}^{p},
\end{aligned}
$$

which yields that $u \geqq 0$ for a.e. $x$ in $\Omega$. Thus, non-trivial critical points of the functional $\Phi_{\lambda}$ are non-negative, non-trivial solutions of problem (1.1)-(1.2). 
The following lemma shows that the functional $\Phi_{\lambda}$ satisfies all of the assumptions of Theorem 3. Then problem (1.1)-(1.2) admits a weak solution $u_{1} \in H$ as a global minimiser and $u_{1} \geqq 0$.

LEMMA 3. The functional $\Phi_{\lambda}$ is bounded from below and satisfies the (PS) condition on $H$.

Proof. By conditions $\left(\mathrm{F}_{1}\right)$ and $\left(\mathrm{F}_{3}\right)$, there exists a constant $C_{\lambda}=C(\lambda)>0$ such that

$$
\lambda F(x, t) \leqq \frac{k_{1} S}{2 p}|t|^{p}+C_{\lambda}
$$

for all $t \in \mathbb{R}$ and a.e. $x \in \Omega$. Hence,

$$
\begin{aligned}
\Phi_{\lambda}(u) & =\int_{\Omega} A(x, \nabla u) \mathrm{d} x-\lambda \int_{\Omega} F(x, u) \mathrm{d} x \\
& \geqq \frac{k_{1}}{p} \int_{\Omega} h_{1}(x)|\nabla u|^{p} \mathrm{~d} x-\int_{\Omega}\left(\frac{k_{1} S}{2 p}|u|^{p}+C_{\lambda}\right) \mathrm{d} x \\
& \geqq \frac{k_{1}}{2 p}\|u\|_{H}^{p}-C_{\lambda}|\Omega|,
\end{aligned}
$$

where $|\Omega|$ denotes the Lebesgue measure of $\Omega$ in $\mathbb{R}^{N}$. Thus, the functional $\Phi_{\lambda}$ is coercive and hence bounded from below on $H$.

Let $\left\{u_{m}\right\}$ be a Palais-Smale sequence in $H$, i.e.

$$
\left|\Phi_{\lambda}\left(u_{m}\right)\right| \leqq c \text { for all } m, \quad \Phi_{\lambda}^{\prime}\left(u_{m}\right) \rightarrow 0 \text { in } H^{\star} .
$$

Since $\Phi_{\lambda}$ is coercive on $H,\left\{u_{m}\right\}$ is bounded in $H$. By Remark 1 (ii), $\left\{u_{m}\right\}$ is bounded in $W_{0}^{1, p}(\Omega)$. It follows that there exists $u \in W_{0}^{1, p}(\Omega)$ such that, passing to a subsequence, still denoted by $\left\{u_{m}\right\}$, it converges weakly to $u$ in $W_{0}^{1, p}(\Omega)$. We shall prove that $\left\{u_{m}\right\}$ converges strongly to $u$ in $H$.

Indeed, we observe by Remark 1(i), Proposition 3(i) and (3.6) that $u \in H$. Hence, $\left\{\left\|u_{m}-u\right\|_{H}\right\}$ is bounded. This and (3.6) imply that $D \Phi_{\lambda}\left(u_{m}\right)\left(u_{m}-u\right)$ converges to 0 as $m \rightarrow \infty$.

Using condition $\left(\mathrm{F}_{1}\right)$ combined with Hölder's inequality we deduce that

$$
\begin{aligned}
0 \leqq \int_{\Omega}\left|f\left(x, u_{m}\right)\right|\left|u_{m}-u\right| \mathrm{d} x & \leqq C \int_{\Omega}\left|u_{m}\right|^{p-1}\left|u_{m}-u\right| \mathrm{d} x \\
& \leqq C\left\|u_{m}\right\|_{L^{p}(\Omega)}^{p-1}\left\|u_{m}-u\right\|_{L^{p}(\Omega)} .
\end{aligned}
$$

Since the embedding $W_{0}^{1, p}(\Omega) \hookrightarrow L^{p}(\Omega)$ is compact, $\left\{u_{m}\right\}$ converges strongly to $u$ in $L^{p}(\Omega)$. Therefore, relation (3.7) implies that

$$
\lim _{m \rightarrow \infty} D I\left(u_{m}\right)\left(u_{m}-u\right)=0 .
$$

Combining relations (3.6) and (3.8) with the fact that

$$
D \Lambda\left(u_{m}\right)\left(u_{m}-u\right)=D \Phi_{\lambda}\left(u_{m}\right)\left(u_{m}-u\right)+D I\left(u_{m}\right)\left(u_{m}-u\right),
$$


we conclude that

$$
\lim _{m \rightarrow \infty} D \Lambda\left(u_{m}\right)\left(u_{m}-u\right)=0
$$

On the other hand, the convex property of functional $\Lambda$ (see Proposition 2(ii)) implies that

$$
\Lambda(u)-\lim _{m \rightarrow \infty} \Lambda\left(u_{m}\right)=\lim _{m \rightarrow \infty}\left(\Lambda(u)-\Lambda\left(u_{m}\right)\right) \geqq \lim _{m \rightarrow \infty} D \Lambda\left(u_{m}\right)\left(u-u_{m}\right)=0 .
$$

Combining this with Proposition 3(i), we have

$$
\lim _{m \rightarrow \infty} \Lambda\left(u_{m}\right)=\Lambda(u)
$$

We now assume by contradiction that $\left\{u_{m}\right\}$ does not converge strongly to $u$ in $H$, and then there exist a constant $\epsilon>0$ and a subsequence $\left\{u_{m_{k}}\right\}$ of $\left\{u_{m}\right\}$ such that $\left\|u_{m_{k}}-u\right\|_{H} \geqq \epsilon$. Using Proposition 2(ii) we get

$$
\frac{1}{2} \Lambda(u)+\frac{1}{2} \Lambda\left(u_{m_{k}}\right)-\Lambda\left(\frac{u_{m_{k}}+u}{2}\right) \geqq k_{0}\left\|u_{m_{k}}-u\right\|_{H}^{p} \geqq k_{0} \epsilon^{p} .
$$

Letting $k \rightarrow \infty$, relation (3.12) gives

$$
\limsup _{k \rightarrow \infty} \Lambda\left(\frac{u_{m_{k}}+u}{2}\right) \leqq \Lambda(u)-k_{0} \epsilon^{p}
$$

We remark that sequence $\left\{\frac{u_{m_{k}}+u}{2}\right\}$ also converges weakly to $u$ in $W_{0}^{1, p}(\Omega)$. So, using Proposition 3(i) again we get

$$
\Lambda(u) \leqq \liminf _{k \rightarrow \infty} \Lambda\left(\frac{u_{m_{k}}+u}{2}\right),
$$

which contradicts (3.13). Therefore, $\left\{u_{m}\right\}$ converges strongly to $u$ in $H$.

LEMMA 4. There exists a positive constant $\bar{\lambda}$ such that for all $\lambda \geqq \bar{\lambda}$, $\inf _{H} \Phi_{\lambda}<0$, and hence $u_{1} \not \equiv 0$, i.e. the solution $u_{1}$ is not trivial.

Proof. Let $\Omega_{0} \subset \Omega$ be a compact subset large enough and a function $\varphi_{0} \in C_{0}^{\infty}(\Omega)$ such that $\varphi_{0}(x)=t_{1}$ in $\Omega_{0}$ and $0 \leqq \varphi_{0}(x) \leqq t_{1}$ in $\Omega \backslash \Omega_{0}$, where $t_{1}$ is chosen as in assumption $\left(\mathrm{F}_{2}\right)$ : then we have

$$
\int_{\Omega} F\left(x, \varphi_{0}\right) \mathrm{d} x \geqq \int_{\Omega_{0}} F\left(x, \varphi_{0}\right) \mathrm{d} x-C t_{1}^{p}\left|\Omega \backslash \Omega_{0}\right|>0 .
$$

Thus, $\Phi_{\lambda}\left(\varphi_{0}\right)<0$ for $\lambda \geqq \bar{\lambda}$ with $\bar{\lambda}$ large enough. This implies that $\inf _{H} \Phi_{\lambda}<0$ and then $\Phi_{\lambda}\left(u_{1}\right)<0$ for $\lambda \geqq \overline{\bar{\lambda}}$, i.e. $u_{1} \neq \equiv$.

In the next part of this paper, we shall show the existence of the second solution $u_{2} \neq u_{1}$ by using the Mountain pass theorem introduced in [3]. To this purpose, we 
first fix $\lambda \geqq \bar{\lambda}$ and set

$$
\widehat{f}(x, t)= \begin{cases}0, & \text { for } t<0, \\ f(x, t) & \text { for } 0 \leqq t \leqq u_{1}(x), \\ f\left(x, u_{1}(x)\right) & \text { for } t>u_{1}(x),\end{cases}
$$

and $\widehat{F}(x, t)=\int_{0}^{t} \widehat{f}(x, s) \mathrm{d} s$. Define the functional $\widehat{\Phi}_{\lambda}: H \rightarrow \mathbb{R}$ by

$$
\widehat{\Phi}_{\lambda}(u)=\int_{\Omega} A(x, \nabla u) \mathrm{d} x-\lambda \int_{\Omega} \widehat{F}(x, u) \mathrm{d} x .
$$

With the same arguments as those used for the functional $\Phi_{\lambda}$, we can show that $\widehat{\Phi}_{\lambda}$ is weakly continuously differentiable on $H$ and

$$
D \widehat{\Phi}_{\lambda}(u)(\varphi)=\int_{\Omega} a(x, \nabla u) \nabla \varphi \mathrm{d} x-\lambda \int_{\Omega} \widehat{f}(x, u) \varphi \mathrm{d} x
$$

for all $u, \varphi \in H$.

LEMMA 5. If $u \in H$ is a critical point of $\widehat{\Phi}_{\lambda}$ then $u \leqq u_{1}$. So, $u$ is a solution of problem (1.1)-(1.2) in the order interval $\left[0, u_{1}\right]$.

Proof. By the definitions of $\Phi_{\lambda}$ and $\widehat{\Phi}_{\lambda}$, if $u$ is a critical point of $\widehat{\Phi}_{\lambda}$ then $u \geqq 0$ as before and by condition $\left(\mathrm{A}_{2}\right)$ we have

$$
\begin{aligned}
0= & \left(D \widehat{\Phi}_{\lambda}^{\prime}(u)-D \Phi_{\lambda}\left(u_{1}\right)\right)\left(\left(u-u_{1}\right)^{+}\right) \\
= & \int_{\Omega}\left(a(x, \nabla u)-a\left(x, \nabla u_{1}\right)\right) \cdot \nabla\left(u-u_{1}\right)^{+} \mathrm{d} x \\
& -\lambda \int_{\Omega}\left(\widehat{f}(x, u)-f\left(x, u_{1}\right)\right)\left(u-u_{1}\right)^{+} \mathrm{d} x \\
= & \int_{\left\{u>u_{1}\right\}}\left(a(x, \nabla u)-a\left(x, \nabla u_{1}\right)\right) \cdot\left(\nabla u-\nabla u_{1}\right) \mathrm{d} x \geqq 0 .
\end{aligned}
$$

According to (3.18) and condition ( $\left.\mathrm{A}_{2}\right)$, the equality holds if and only if $\nabla u=\nabla u_{1}$. It follows that $\nabla u(x)=\nabla u_{1}(x)$ for all $x \in \Omega_{1}:=\left\{y \in \Omega: u(y)>u_{1}(y)\right\}$. Hence,

$$
\int_{\Omega_{1}}\left|\nabla\left(u-u_{1}\right)\right|^{p} \mathrm{~d} x=0 \text { and thus } \int_{\Omega}\left|\nabla\left(u-u_{1}\right)^{+}\right|^{p} \mathrm{~d} x=0 .
$$

Combining this with Remark 1(ii), we conclude that $\left\|\left(u-u_{1}\right)^{+}\right\|_{L^{p}(\Omega)}=0$ and then $\left(u-u_{1}\right)^{+}=0$ in $\Omega$, that is, $u \leqq u_{1}$ in $\Omega$.

LEMMA 6. There exist a constant $\rho \in\left(0,\left\|u_{1}\right\|_{H}\right)$ and a constant $\alpha>0$ such that $\widehat{\Phi}_{\lambda}(u) \geqq \alpha$ for all $u \in H$ with $\|u\|_{H}=\rho$.

Proof. We set $\Omega_{u}=\left\{x \in \Omega: u(x)>\min \left\{u_{1}(x), t_{0}\right\}\right\}$, where $t_{0}$ is given as in $\left(\mathrm{F}_{2}\right)$. Then, by (3.16) and condition $\left(\mathrm{F}_{1}\right)$, we have $\widehat{F}(x, u(x)) \leqq 0$ on $\Omega \backslash \Omega_{u}$. Hence,

$$
\widehat{\Phi}_{\lambda}(u) \geqq k_{1}\|u\|_{H}^{p}-\lambda \int_{\Omega_{u}} \widehat{F}(x, u) \mathrm{d} x .
$$


Using $\left(\mathrm{F}_{1}\right)$, Hölder's inequality and Remark 1(ii), we get

$$
\int_{\Omega_{u}} \widehat{F}(x, u) \mathrm{d} x \leqq C \int_{\Omega_{u}}|u|^{p} \mathrm{~d} x \leqq C\left|\Omega_{u}\right|^{1-\frac{p}{q}}\|u\|_{H}^{p},
$$

where $q=\frac{N p}{N-p}$. Therefore,

$$
\widehat{\Phi}_{\lambda}(u) \geqq\|u\|_{H}^{p}\left(k_{1}-\lambda C\left|\Omega_{u}\right|^{1-\frac{p}{q}}\right) .
$$

In order to prove Lemma 6 , it is enough to show that $\left|\Omega_{u}\right| \rightarrow 0$ as $\|u\|_{H} \rightarrow 0$. Indeed, let $\epsilon>0$ be arbitrary; we choose $\Omega_{\epsilon} \subset \Omega$ a compact subset, large enough such that $\left|\Omega \backslash \Omega_{\epsilon}\right|<\epsilon$, and denote by $\Omega_{u, \epsilon}:=\Omega_{u} \cap \Omega_{\epsilon}$. Then it is clear that

$$
\|u\|_{H}^{p} \geqq\|u\|^{p} \geqq \int_{\Omega}|u|^{p} \mathrm{~d} x \geqq \int_{\Omega_{u, \epsilon}} u^{p} \mathrm{~d} x \geqq l^{p}\left|\Omega_{u, \epsilon}\right|,
$$

where $l=\min \left\{\min u_{1}\left(\Omega_{\epsilon}\right), t_{0}\right\}$. Letting $\|u\|_{H} \rightarrow 0$ we deduce that $\left|\Omega_{u, \epsilon}\right| \rightarrow 0$. Since $\Omega_{u} \subset \Omega_{u, \epsilon} \cup \Omega \backslash \Omega_{\epsilon}$ we have $\left|\Omega_{u}\right| \leqq\left|\Omega_{u, \epsilon}\right|+\epsilon$ with $\epsilon>0$ as arbitrary. Thus, $\left|\Omega_{u}\right| \rightarrow 0$ as $\|u\|_{H} \rightarrow 0$.

\section{LEMMA 7. Functional $\widehat{\Phi}_{\lambda}$ satisfies the (PS) condition on $H$.}

Proof. We observe by (3.21) that $\widehat{\Phi}_{\lambda}$ is coercive. Therefore, every Palais-Smale sequence of $\widehat{\Phi}_{\lambda}$ is bounded in $H$. We follow the method as those used in the proof of Lemma 3. It can be shown that the functional $\widehat{\Phi}_{\lambda}$ satisfies the (PS) condition on $H$.

Proof of Theorem 2. By Lemmas 2-4, using Theorem 3, problem (1.1)-(1.2) admits a non-negative, non-trivial weak solution $u_{1}$. Setting

$$
c:=\inf _{\gamma \in \Gamma} \max _{u \in \gamma([0,1])} \widehat{\Phi}_{\lambda}(u),
$$

where $\Gamma:=\left\{\gamma \in C([0,1], H): \gamma(0)=0, \gamma(1)=u_{1}\right\}$, Lemmas 6-7 show that all of the assumptions of Proposition 1 are fulfilled, $\widehat{\Phi}_{\lambda}\left(u_{1}\right)=\Phi_{\lambda}\left(u_{1}\right)<0$ and $\left\|u_{1}\right\|_{H}>\rho$. Then, $c>0$ is a critical value of $\widehat{\Phi}_{\lambda}$, i.e. there exists $u_{2} \in H$ such that $D \widehat{\Phi}_{\lambda}\left(u_{2}\right)(\varphi)=0$ for all $\varphi \in H$ and $\widehat{\Phi}_{\lambda}\left(u_{2}\right)=c>0$. By Lemma $5,0 \leqq u_{2} \leqq u_{1}$ in $\Omega$. Therefore, using (3.16) some simple computations give us

$$
\widehat{\Phi}_{\lambda}\left(u_{2}\right)=\Phi_{\lambda}\left(u_{2}\right), \quad D \widehat{\Phi}_{\lambda}\left(u_{2}\right)(\varphi)=D \Phi_{\lambda}\left(u_{2}\right)(\varphi) \text { for all } \varphi \in H .
$$

By Remark 1(iii), we conclude that $u_{2}$ is a weak solution of problem (1.1)-(1.2). Furthermore, $\Phi_{\lambda}\left(u_{2}\right)=c>0>\Phi_{\lambda}\left(u_{1}\right)$. Thus, $u_{2}$ is not trivial and $u_{2} \neq u_{1}$. The proof of Theorem 2 is now complete.

\section{REFERENCES}

1. A. Ambrosetti and P. H. Rabinowitz, Dual variational methods in critical points theory and applications, J. Funct. Anal. 4 (1973), 349-381.

2. P. De Nápoli and M. C. Mariani, Mountain pass solutions to equations of $p$-Laplacian type, Nonlinear Anal. 54 (2003), 1205-1219. $420-440$.

3. D. M. Duc, Nonlinear singular elliptic equations, J. Lond. Math. Soc. 40(2) (1989), 
4. D. M. Duc and N. T. Vu, Non-uniformly elliptic equations of $p$-Laplacian type, Nonlinear Anal. 61 (2005), 1483-1495.

5. I. Ekeland, On the variational principle, J. Math. Anal. Appl. 47 (1974), 324-353.

6. L. Gasiński and N. S. Papageorgiou, Nonsmooth critical point theory and nonlinear boundary value problems. Series in Mathematical Analysis and Applications, 8. Chapman \& Hall/CRC, Boca Raton, FL, 2005.

7. M. Mihăilescu and V. Rădulescu, A multiplicity result for a nonlinear degenerate problem arising in the theory of electrorheological fluids, Proc. R. Soc. Lond. Ser. A 462 (2006), 2625-2641.

8. M. Mihăilescu, Existence and multiplicity of weak solutions for a class of denegerate nonlinear elliptic equations, Boundary Value Probl. (2006), Art. ID 41295, 17 pp.

9. Q.-A. Ngô, and H. Q. Toan, Existence of solutions for a resonant problem under Landesman-Lazer conditions, Electron. J. Diff. Eqns. 2008 (98) (2008), 1-10.

10. K. Perera, Multiple positive solutions for a class of quasilinear elliptic boundary-value problems, Electron. J. Differential Equations 7 (2003), 1-5.

11. M. Struwe, Variational Methods: Applications to nonlinear partial differential equations and Hamiltonian systems, 4 ed. (Springer-Verlag, Berlin, 2008).

12. H. Q. Toan and Q.-A. Ngô, Multiplicity of weak solutions for a class of non-uniformly elliptic equations of $p$-Laplacian type, Nonlinear Anal. 70 (2009), 1536-1546.

13. N. T. Vu, Mountain pass theorem and non-uniformly elliptic equations, Vietnam J. Math 33 (4) (2005), 391-408. 